\title{
Anomalous origin of left coronary artery from the pulmonary artery in a 60-year-old woman
}

Yinsu Zhu, MD, Lijun Tang, MD, PhD, and Yi Xu, MD

A 60-year-old mother of 2 children who had been doing heavy manual work for years reported a history longer than 1 year of dyspnea, palpitations, and chest pain. She had no coronary risk factors or history of cardiovascular disease. Her serial cardiac enzyme results were negative, and the electrocardiogram showed nonspecific ST-T changes.

Coronary computed tomography angiography revealed an enormously dilated, tortuous, and dominant right coronary artery originating from the right coronary cusp (Figure 1, $A$ ), with a large number of collateral vessels feeding the left coronary system (Figure 2). The left coronary artery (LCA) arose from the main pulmonary artery immediately adjacent to the left sinus of Valsalva (Figure 1). The left anterior descending artery and left circumflex artery were branching from left main coronary artery. The left coronary system, including left main coronary artery, left anterior descending artery, left circumflex artery, and their diagonal and obtuse branches, were dilated and tortuous. A diagnosis of

From the Department of Radiology, the First Affiliated Hospital of Nanjing Medical University, Nanjing, People's Republic of China.

Supported by a project funded by the Priority Academic Program Development of Jiangsu Higher Education Institutions (JX10231801), National Natural Science Foundation of China (No. 81101104), and Natural Science Foundation of Jiangsu Province (BK2012743).

Disclosures: Authors have nothing to disclose with regard to commercial support. Y.Z. and L.T. contributed equally to this work.

Received for publication Sept 28, 2013; accepted for publication Oct 4, 2013; available ahead of print Nov 22, 2013

Address for reprints: Yinsu Zhu, MD, Department of Radiology, the First Affiliated Hospital of Nanjing Medical University, 300 Guangzhou Rd, Nanjing, Jiangsu 210029, People's Republic of China (E-mail: zhuyinsu1982@gmail.com).

J Thorac Cardiovasc Surg 2014;147:1706-7

$0022-5223 / \$ 36.00$

Copyright (C) 2014 by The American Association for Thoracic Surgery

http://dx.doi.org/10.1016/j.jtcvs.2013.10.017 anomalous origin of the LCA from the pulmonary artery (ALCAPA) syndrome was confirmed. The left ventricular ejection fraction, as evaluated with cine cardiovascular magnetic resonance, was a little decreased $(50 \%)$, with anterior, anterolateral, and anteroseptal wall hypokinesia and mild mitral regurgitation. The ischemic area characteristically involved a subendocardial layer of the basal to midseptal, anterior, and apical anteroseptal segments, as confirmed with late gadolinium enhancement (LGE) cardiovascular magnetic resonance (Figure 3).

Because of the patient's advanced age and her family's request, she was discharged home on medication, including aspirin (INN acetylsalicylic acid) and losartan potassium (INN losartan) a few days later. After 9 months of followup, she remains free of severe symptoms.

\section{DISCUSSION}

ALCAPA is a rare congenital anomaly, also known as Bland-White-Garland syndrome, and accounts for about $0.25 \%$ to $0.5 \%$ of congenital heart defects. ${ }^{1}$ Symptoms usually occur in infants 1 to 2 months after birth because of left-to-right shunting from the higher pressure left coronary arterial system to the lower pressure pulmonary arterial system. The patients may survive into childhood to adulthood, however, with clinical presentations varying from symptomatic chronic mitral insufficiency to global ischemic cardiomyopathy. ${ }^{2,3}$ Factors that may lead to survival beyond infancy include the development of collateral circulation from the right coronary artery to the LCA and reversal of flow from the pulmonary artery into the LCA. Our patient's lack of symptoms for many years until presentation may be related to extensive collateral

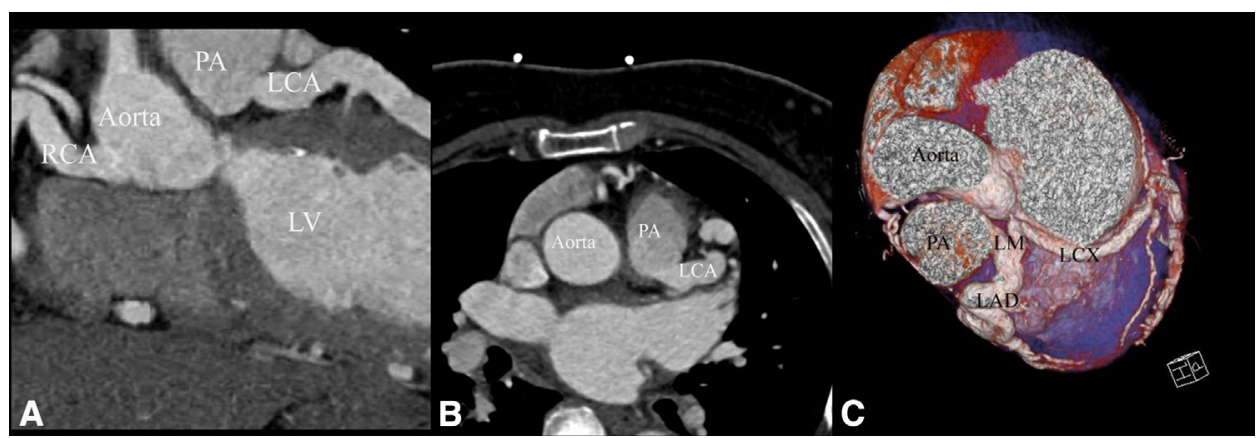

FIGURE 1. A-C, Coronary computed tomography angiography demonstrates the origin of the dilated left coronary artery $(L C A)$ from the pulmonary arterial $(P A)$ trunk. The right coronary artery $(R C A)$ originated from the right coronary cusp. $L V$, Left ventricle; $L M$, left main artery; $L C X$, left circumflex coronary artery; $L A D$, left anterior descending coronary artery. 


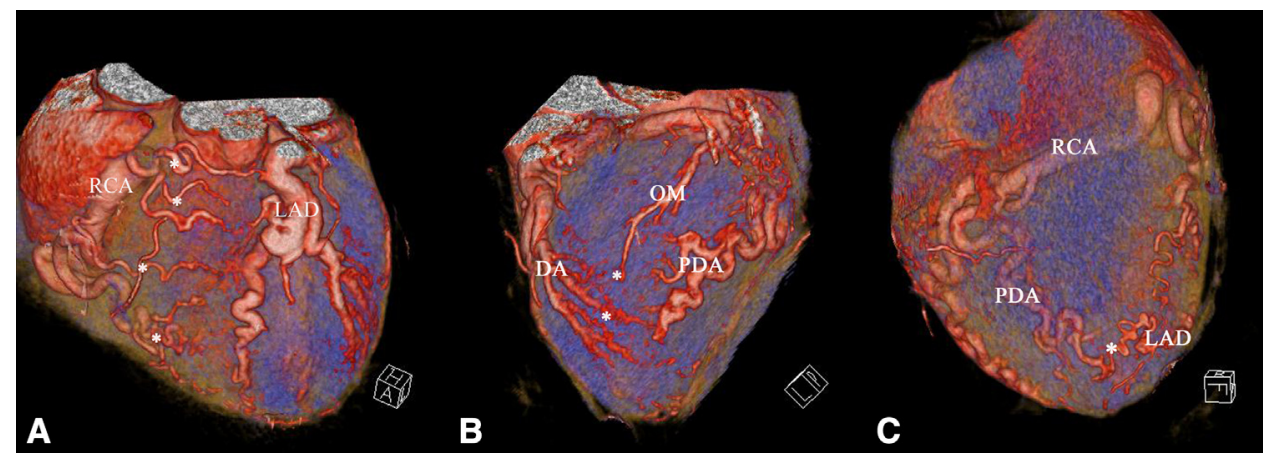

FIGURE 2. A-C, Volumetric rendering images demonstrate the dilated right coronary artery $(R C A)$ and left coronary artery system. Extensive collateral vessels between them are presented crossing the free wall of the right ventricle, the inferolateral wall of the left ventricle, and the apex of the heart (within asterisks). LAD, Left anterior descending coronary artery; OM, obtuse marginal branch; $D A$, diagonal branch; $P D A$, posterior descending artery.

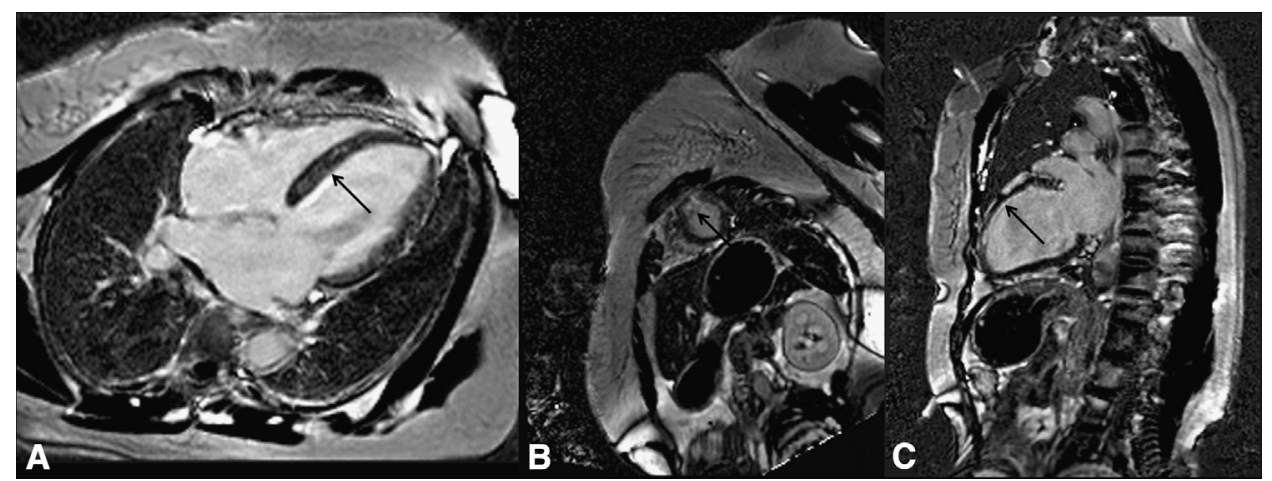

FIGURE 3. A-C, Late gadolinium enhancement images from cardiovascular magnetic resonance show varying degrees of subendocardial late gadolinium enhancement in the basal to midseptal, anterior, and apical anteroseptal segments (black arrows).

vessels between the LCA and right coronary artery, which provided enough oxygenated blood to the myocardium.

In our patient, coronary computed tomography angiography provided direct visualization of the LCA arising from the main pulmonary artery, which is the diagnostic hallmark of ALCAPA syndrome. It also revealed a dilated and tortuous right coronary artery and dilated intercoronary collateral arteries along the epicardial surface of the heart. In addition to coronary computed tomography angiography, cardiovascular magnetic resonance is a good, noninvasive, radiation-free investigation in the preoperative and postoperative evaluation of ALCAPA, not only assessing left ventricular systolic function but also helping to determine myocardial ischemia and viability.

\section{References}

1. Pfannschmidt J, Ruskowski H, de Vivie ER. [Bland-White-Garland syndrome. Clinical aspects, diagnosis, therapy]. Klin Padiatr. 1992;204:328-34. German.

2. Yau JM, Singh R, Halpern EJ, Fischman D. Anomalous origin of the left coronary artery from the pulmonary artery in adults: a comprehensive review of 151 adult cases and a new diagnosis in a 53-year-old woman. Clin Cardiol. 2011;34:204-10.

3. Separham A, Aliakbarzadeh P. Anomalous left coronary artery from the pulmonary artery presenting with aborted sudden death in an octogenarian: a case report. J Med Case Rep. 2012;6:12. 